\title{
The Italian Twin Register: New Cohorts and Tools, Current Projects and Future Perspectives of a Developing Resource
}

\author{
Corrado Fagnani, Sonia Brescianini, Rodolfo Cotichini, Cristina D'lppolito, Tatjana Dukic, Lorenzo \\ Giannantonio, Emanuela Medda, Lorenza Nisticò, Valeria Patriarca, Simonetta Pulciani, Daniela Rotondi, \\ Virgilia Toccaceli, and Maria Antonietta Stazi \\ National Center for Epidemiology, Surveillance and Health Promotion, Istituto Superiore di Sanità, Rome, Italy
}

Sinte ince its start as a database of 'possible twins', the Italian Twin Register has developed remarkably in terms of twin approach and recruitment, data-management tools, the cohorts enrolled, and the breadth of information gathered, making the Italian Twin Register a valuable resource for genetic epidemiological research. The Italian Twin Register is a random population of twins at both the national level and within targeted geographical areas or birth cohorts. Further, the Register is linked with disease records and has recently implemented a web-based method for volunteer twin recruitment specifically designed to promote the Register and to disseminate information on genetic epidemiology. To date, approximately 9000 twins have joined the Italian Twin Register, the majority of whom (approximately 70\%) represent young adults aged 20 at time of enrolment. Although the total number of twins recruited to date is far below the expected figure initially predicted, the newly established standardized procedures guarantee an increase of around 2000 twins each year. Following the collaboration between the Italian Twin Register and the main Italian nonprofit association for blood donors, twin DNA sampling and storage has recently accelerated contributing to the large amount of phenotypic data collected. The Italian Twin Register is currently involved in both population and clinical based studies on various complex phenotypes and diseases, some conducted within large European consortia.

In 2001 the Italian government, through the Ministry of Health, funded a broad research program on twin studies, including the establishment of a national twin register at the Italian National Institute of Health (Istituto Superiore di Sanità, ISS). The Italian Twin Register (ITR) started as a database of 'possible twins'. This database was made feasible by the 'fiscal code', an alphanumeric identification code that was introduced in 1976 by the Ministry of Finance, which contains demographic information on any single person residing in Italy. About 650,000 pairs of possible twins, born before 31 December 1996, were identified by their fiscal code that indicated they shared the same surname and date and place of birth. Within the database, an average of $40 \%$ excess of pseudotwins, mainly localized in the elderly, was estimated (Stazi et al., 2002).

For the enrolment of twins in the ITR, two main approaches are followed to assess the confirmation of twin status: (1) a questionnaire is mailed to possible twins from the general population, both at national level and within targeted geographical areas or birth cohorts, and (2) potential twin patients are detected by linking the database of possible twins with disease records. The first approach corresponds to the random population ascertainment of twins, and is appropriate to study either quantitative traits or common diseases; the second method, providing that confidentiality and privacy issues are met, enables the collection of twin pairs in which at least one member is affected, and ensures a gain in terms of statistical power when relatively rare disorders are to be investigated.

Recently, a web site of the ITR (www.gemelli.iss.it) has been designed in order to optimally promote the register and its scientific value, and better disseminate information concerning genetic epidemiology; this also allowed the implementation of a web-based method for the volunteer twin recruitment.

To date, approximately 9000 twins have joined the ITR; although the total number of twins recruited to date is far below the expected number of twins forecasted in the early stages, enrolment follows standardized procedures, with an increase of around 2000 twins each year. Table 1 shows the total numbers of twin pairs recruited by year of birth, gender and zygosity.

Zygosity is largely determined by means of standard questions on physical similarity and frequency of

Received 28 July, 2006; accepted 29 August, 2006.

Address for correspondence: Corrado Fagnani, National Center for Epidemiology, Surveillance and Health Promotion, Istituto Superiore di Sanità,00161 Rome, Italy.E-mail: corrado.fagnani@iss.it 
Table 1

Distribution of the Twin Pairs According to Year of Birth, Zygosity and Gender

\begin{tabular}{|c|c|c|c|c|c|c|c|}
\hline & \multicolumn{2}{|c|}{ Monozygotic } & \multicolumn{3}{|c|}{ Dizygotic } & \multirow[t]{2}{*}{ Not definable } & \multirow[t]{2}{*}{ Total } \\
\hline & Males & Females & Males & Females & Opposite-sex & & \\
\hline Year of birth & Pairs \% & Pairs \% & Pairs \% & Pairs \% & Pairs \% & Pairs \% & Pairs \% \\
\hline Elderly (1940 and earlier) & 6514.9 & 7817.9 & 7316.7 & 7517.2 & 13230.3 & 133.0 & 436100.0 \\
\hline Adults (1980-1941) & 4116.3 & 6525.8 & 3614.3 & 3714.7 & 7228.6 & 10.4 & 252100.0 \\
\hline Young adults (1988-1981) & 54517.5 & 80825.9 & 38412.3 & 51616.6 & 81226.1 & 511.6 & 3116100.0 \\
\hline Children \& teenagers (1996-1989) & 11120.9 & 9217.4 & 7313.8 & 9918.7 & 15429.1 & 10.2 & 530100.0 \\
\hline Total & 76217.6 & 104324.1 & 56613.1 & 72716.8 & 117027.0 & 661.5 & 4334100.0 \\
\hline
\end{tabular}

confusion of the twins by family members, teachers and friends. However, the establishment of a biological bank at the ISS with the storage of twins' DNA will enable assessment of the reliability of the questionnaire method for classifying zygosity.

The ITR is currently involved in both clinical and population-based studies on various complex phenotypes and diseases, and is part of European consortia for genetic epidemiological research.

\section{Population-Based Studies}

Twins from the general population are participating in studies mainly focused on behavioral research (children, adolescents, and young adults), cognitive functioning, and eye disease (elderly). Young adult twins, who represent the largest cohort recruited in the ITR, are also participating in the GenomEUtwin project (www.genomeutwin.org), supported by the European Commission under the 'Quality of Life and Management of the Living Resources' program of the 5th Framework Programme (FP), for the study of stature and body mass index. Twin donors are actively recruited all over the country through the main Italian nonprofit association for blood donors (AVIS).

\section{Children and Adolescents}

Three hundred and seventy-eight twin pairs, aged 8 to 17 years, residing in the northern provinces of Milano and Lecco, participated in a study on anxiety disorders that investigated the role of genetic and environmental factors in determining interindividual variation of anxiety dimensions as measured by the Italian version of the Screen for Child Anxiety-Related Emotional Disorders questionnaire (SCARED; Ogliari et al., 2006). An exploratory factor analysis (EFA) provided four distinct factors that considerably overlapped with the original SCARED subscales of generalized anxiety disorder (GAD), panic disorder (PD), social phobia disorder (SP) and separation anxiety disorder (SAD).

The individual scores in each EFA-derived empirical factor were analyzed by standard univariate twin modeling. Moderate to high heritability (ranging from $53 \%$ to $60 \%$ ), without age or sex differences, emerged for all dimensions, with the exception of GAD for which an age effect was found (Table 2). This study is part of an ongoing project regarding health-related characteristics and behavior in Italian children. The Italian versions of the International Study of Asthma and Allergies in Childhood (ISAAC) and of the Child Behavior Check List (CBCL) questionnaire were administered to the same twin sample; at present, allergic diseases and internalizing and externalizing behavior problems are being investigated.

\section{Young Adults}

Three thousands twin pairs, aged 21 to 23 years, are taking part in a study which investigates the role of genetic and environmental factors in determining interindividual variation in emotional regulation. This study is part of an ongoing project regarding healthrelated characteristics and behaviors in young Italian adults. It focuses on individual difference factors such as attachment insecurity and alexithymia, which have been found to be associated with poor mental and physical health. The validated Italian versions of the Experiences in Close Relationships (ECR) questionnaire and the 20-item Toronto Alexithymia Scale (TAS-20) are being administered to this twin sample. These instruments provide valid and reliable assessments of attachment-related anxiety and avoidance (ECR), difficulty recognizing and describing feelings, and difficulty with externally oriented thinking (TAS-20). The results of this study are expected by spring 2007 .

Another young adult twin population encompasses predominantly consecutive year-of-birth cohorts of twin pairs aged 20 at the time of enrolment: the 1983, 1984, and 1985 cohorts were recruited in 2003, 2004, and 2005 respectively, and the enrolment of the 1986 cohort is underway. These cohorts have several advantages: the twins' age allows personal consent for enrolment (very often they still live together and thus the first mail contact is cheaper), their parents are often close by and early life events can easily be investigated. The 20-year-old twins are to date really the only healthy population recruited at national level. These cohorts were included in the GenomEUtwin project giving the highest estimate values for heritability of height and body mass index among the 
European cohorts (Table 2; Schousboe et al., 2003; Silventoinen et al., 2003).

A subset of this cohort (young adult twins residing in Rome) are also participating to the EuroClot Project (http://www.twin-research.ac.uk/euroclot.html). This project, supported by the European Commission's FP6, focuses on uncovering the genes that control the end-stage of the coagulation process that leads directly to the production of the thrombus causing vascular obstruction and tissue death.

\section{Elderly}

Despite the long-standing Italian tradition of twin studies, there are no reports in the literature on the contribution of genetic influences to cognitive and physical performance in elderly Italian twins. Twin pairs born between 1926 and 1940, residing in Rome and in Latina, were identified in the ITR using the personal identification number. Out of 436 pairs recruited in the health survey, 160 pairs attended a clinical examination aimed to assess the cognitive and physical functions of the twins. Cognitive performance was evaluated on a battery of neuropsychological tests and the scores of each assessment instrument were adjusted for age and education; physical function was also investigated (lower extremity function was measured by the Established Populations for the Epidemiologic Studies of the Elderly battery). Zygosity was assessed by a twin similarity questionnaire. Twin concordances for cognitive performance and physical activity were assessed using twin correlations. Estimates of the proportions of variance due to additive genetic influence $(\mathrm{A})$, shared environmental effects (C) or genetic dominance effects (D), and nonshared environmental factors (E) were calculated. A preliminary analysis shows the greater importance of genetic factors in different areas of cognition: Mini Mental State Examination $(\mathrm{A}=55 \%)$, Raven $(\mathrm{A}=56 \%)$, Attentional Matrix $(\mathrm{A}=79 \%)$, Copying Drawing $(\mathrm{A}=$ $69 \%)$, and Story Recall $(\mathrm{A}=54 \%)$. The study is still ongoing: more than one hundred twin pairs are to be enrolled in the future and all participants are scheduled for a follow-up survey.

To date 120 twin pairs aged 50 to 85 years ( 40 monozygotic [MZ] and 80 dizygotic [DZ]) have been enrolled in an age-related cataract study in the city of Parma, from a long-lasting collaboration with the ophthalmology department of the local university (The Italian-American Cataract Study Group, 1991).

At baseline, the participants receive ocular examination and photographic lens grading; the type and severity of the opacities are assessed by slit-lamp and retro-illumination lens photographs.

Detailed information on medical history is collected, as well as DNA samples and measurement of plasma and serum biochemical levels. This study aims to enrol 300 pairs in the province of Parma to evaluate the heritability of cataracts in an area where intensive information on environmental risk factors for age-related eye diseases already exists.
The ITR is also involved in the Genetics of Healthy Aging project (GEHA, www.geha.unibo.it). This project, supported by the European Commission's FP6, aims to study the genetic determinants of human longevity. Within this project, the ITR is recruiting MZ and DZ twin pairs older than 90, residing in the province of Rome. Genome scans will be conducted on the DZ pairs who will take part in a linkage study with the 2500 nonagenarian sibling pairs recruited in 11 European Countries, and mitochondrial DNA germline variants and mutations will be investigated related to the health status of the MZ pairs.

\section{Linkage of the ITR with Disease Registers}

One of the main strengths of the ITR is the ability to perform record linkages between personal twin data and disease data from registers held in clinical centers in order to identify twins affected by a certain disease in a multicenter study setting.

Two clinically based studies on autoimmune diseases in Italian twins, both performed using the above procedure, have recently been published. One study focuses on celiac disease (CD; Nisticò et al., 2006) and aims to estimate: (1) disease concordance rates by zygosity and HLA genotypes, (2) progression rates to the disease, and (3) heritability. The membership lists of the Italian Celiac Disease Association (AIC), including CD patients from Southern (Campania, Basilicata, Puglia, Calabria, Sicilia) and Northern (Piemonte) Italy regions were linked with the database of the ITR. Eighty-one twin pairs were identified, of which 73 entered the study.

Concordance rates were significantly higher in $\mathrm{MZ}$ (83.3\% probandwise, $71.4 \%$ pairwise) than in DZ pairs $(16.7 \%$ probandwise, $9.1 \%$ pairwise), suggesting a substantial genetic contribution in CD etiology. After stratifying the sample into four HLA genotype groups with decreasing disease risk, in each stratum concordance was higher in MZ pairs compared to DZ pairs sharing identical HLA chromosomes; this was consistent with the existence of non-HLA loci associated with or linked to $\mathrm{CD}$ in the Italian population. In $90 \%$ of concordant pairs, the discordance time (i.e., the time between the diagnosis in the proband and that in the co-twin) was less than 2 years; furthermore, $\mathrm{MZ}$ and $\mathrm{DZ}$ co-twins had respectively $70 \%$ and $9 \%$ cumulative probability to be diagnosed within 5 years of the diagnosis in the proband. Genetic modeling provided a heritability estimate of $57 \%$ and $87 \%$ under an ACE model assuming a CD population prevalence of $1 / 1000$ and $1 / 91$, respectively (Table 2 ).

The other recent clinically based study regards multiple sclerosis (MS) in continental Italy and Sardinia, which are medium and high prevalence areas, respectively (Ristori et al., 2006). For this study, the database of the ITR was matched with the records of Italian MS clinics and those of the Italian MS Society (Associazione Italiana Sclerosi Multipla), leading to an ascertainment of 216 twin pairs from a 
Table 2

Recently Published Twin Studies Using Data From the Italian Twin Register

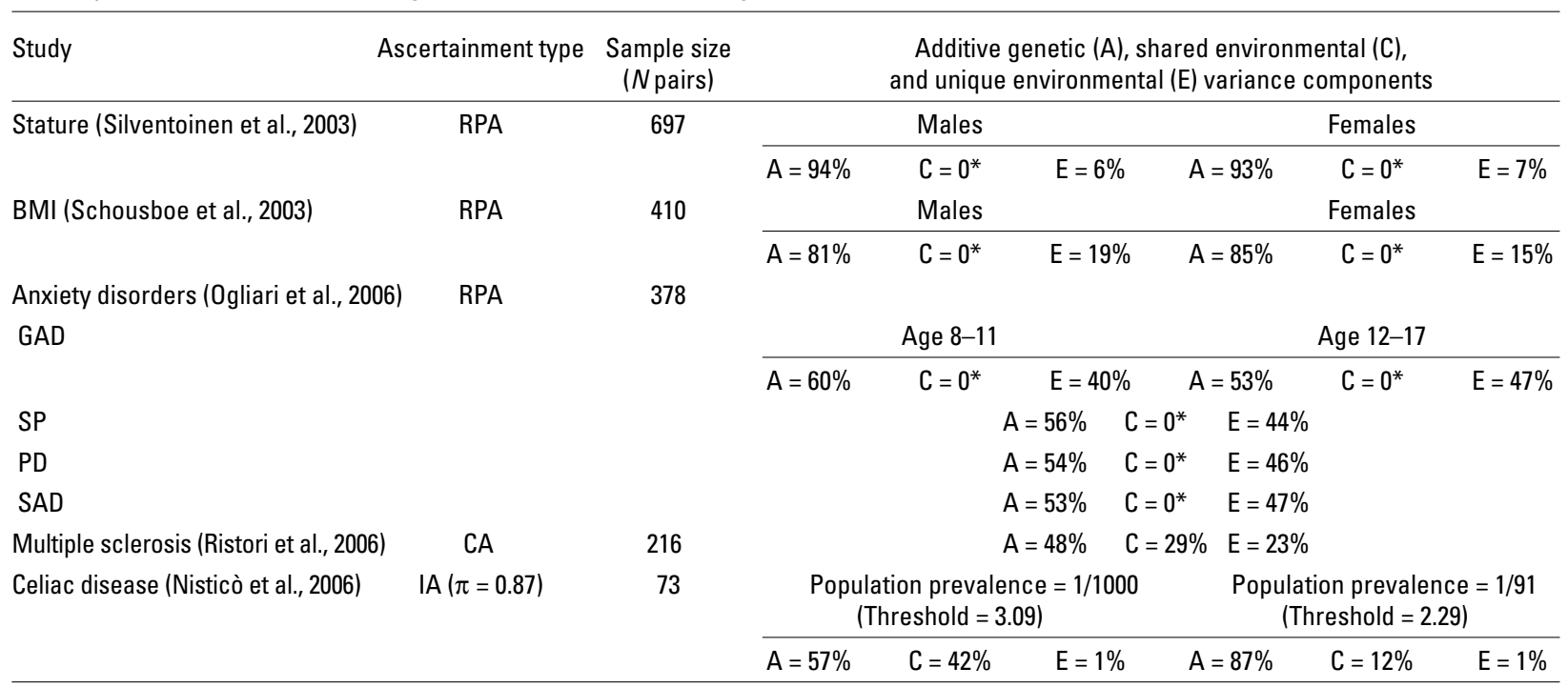

Note: $\mathrm{RPA}=$ random population ascertainment; $\mathrm{CA}=$ complete ascertainment; $\mathrm{IA}=$ incomplete ascertainment; $\pi=$ ascertainment probability; $\mathrm{BMI}=$ body mass index;

$\mathrm{GAD}=$ generalized anxiety disorder; $\mathrm{SP}=$ social phobia disorder; $\mathrm{PD}=$ panic disorder; $\mathrm{SAD}=$ separation anxiety disorder. $0^{*}=$ fixed to zero in the best fitting model.

total of 34,549 patients. This gave a twin rate of $0.62 \%$ among MS patients, significantly less than that observed in the general population $(1 \%)$; the underrepresentation of twins among MS patients drew attention to protective factors shared by twins that may influence disease susceptibility.

In continental Italy, probandwise concordance was $14.5 \%$ for $\mathrm{MZ}$ and $4.0 \%$ for DZ twins. In Sardinia, MZ concordance was $22.2 \%$ probandwise, but no concordant DZ pairs were identified. Genetic modeling of data from continental Italy gave a heritability estimate of $48 \%$, with an environmental contribution of $29 \%$ for shared and $23 \%$ for unique environmental factors (Table 2).

A subsample of MZ discordant pairs is currently being analyzed at the molecular level to detect possible epigenetic differences between the affected twins and their healthy co-twins.

The procedure described above was quite efficient in detecting twins within a list of patients with a specific disease. Since 2003, when individual privacy protection for health data in Italy was legally established (legislative decree n. 196; 30 June 2003), these studies have been faced with ethical concerns mainly around informed consent, privacy, confidentiality and security data management. A new procedure has had to be introduced (see paragraph on key privacy and ethical issues), allowing linkage within the new rules.

Other studies on autoimmune diseases are now linking the ITR to the disease registers. The Italian Society of Diabetes and Endocrinology Pediatricians is collaborating with the ITR in a study at national level aimed at detecting twins with type 1 diabetes. Several clinics have already matched their lists of patients to the twin register and are recruiting nonaffected sib- lings into the study. A similar collaboration has been set up with the Italian network for inflammatory bowel disease, looking for twins with Crohn's disease, and with a consortium of clinicians interested in the genetic factors of systemic lupus erythematosus (Priori et al., 2003).

The main focus of all these studies on autoimmune diseases is to collect $\mathrm{MZ}$ pairs discordant for the various autoimmune pathologies under investigation. MZ discordant pairs are a new 'tool' in genetic epidemiology (Fraga et al., 2005): examining different gene expression profiles (Sarkijarvi et al., 2006), locus-specific DNA methylation patterns, asymmetric mitochondria transmission or X-chromosome inactivation in female pairs in these genetically homogeneous individuals provides an exceptional opportunity to investigate the genetic nature of autoimmune diseases.

\section{ITR Data Acquisition and Storage}

Twin data are collected either by mail questionnaires, personal interviews or web-based forms.

An optical character recognition system is currently utilized for processing all the nonelectronic ITR documents (enrolment, health status, and study-specific questionnaires). The applied software is composed of different phases for designing, scanning, reading, verifying, and storing questionnaires. For each group of investigated subjects, a tailored questionnaire - or set of questionnaires - is created. A bar-code containing the identification number of the pair has been added to all forms except for the enrolment ones, which already include a clear identification for each subject. The design of these ad hoc forms includes the setting of specific control scripts to, for 
example, acquire values within appropriate ranges, skip logical missing items, or calculate specific formulas. In order to optimize data acquisition, the scanner used was able to process a large number of forms in a short time, with different sizes of paper, and to scan both sides automatically.

After the scanning step, any ambiguously read data are verified and validated by an operator.

For each form, both image and data are stored separately. The storage of images enables the recovery of an original document - or groups of documents using selected searching keys (dates, specific values, geographical data, and so on).

The web registration site (Twin On-Line) is based on a standard Apache/Php/MySQL GNU/GPL suite of scripts and tables. In order to preserve twins' privacy, the MySQL database keeps personal and scientific data in separate autonomous tables. As a result, it is easy to grant different levels of access to staff; for instance, the statistic staff are granted access to science data, administrative staff to personal and contact data, with only the system administrator able to link the data from the two tables. The front-end access system is based on the Php sessions/cookies architecture, and is designed in order to allow registered twins the possibility to view and modify only their data. The back-end administration access system is based on the HTTP Authentication Php module. Finally, directories have been designed in order to keep the most critical scripts (e.g., scripts containing access information) in an area which is outside the DocumentRoot tree, thus making them intrinsically invisible to HTTP clients.

A web-based questionnaire was sent by email in May 2006 to 800 twins (young adults) in order to test their compliance to the procedure. Opinions about mailing saliva for DNA banking, awareness on scientific studies purposes, privacy issues and attitude to voluntary service were investigated.

About $80 \%$ of the responses arrived in the first week, indicating the method's effectiveness.

\section{The TWINNET Database}

One of the major tasks of the GenomEUtwin project was to share standard operating procedures on data management within the network of the participating twin registers and to eventually implement a 'common' database. Data managing in GenomEUtwin is based on a federated approach: each center stores its own data, but for the GenomEUtwin user, all data appears to be stored in one place, accessed by a hub. A common format and variable standard for phenotypes (current GenomEUtwin_Data_Format v4.0) has been prepared by the data core members. The aim of the format and variable standard is to facilitate work in the planning, analysis and archiving stage of GenomEUtwin, to increase accuracy and comprehensibility of data and thereby enable a smooth transfer of datasets.
Data are loaded in a premade database in MySQL 5.0 , prepared by the group responsible for data integration. To date, we are following the database schema described in Weight and Height Federated Database, Version 1.2. The variables (columns) and values are based on variable formats and standards described in GenomEUtwin_Data_Format v4.0. The schema contains tables for subject, weight, height, alcohol and tobacco usage and socioeconomic status. In addition, the database has prepopulated auxiliary tables containing information on used codes. An important issue regarding data harmonization is the EUid number, which is an identification number for the individuals whose data is stored in the database. The number is unique and each center is responsible for creating an EUid number for its individuals. The EUid number consists of four parts: country, randomized number, identification number and check sum.

\section{DNA Collection and Banking}

As ITR is not in a hospital setting, all the procedures requiring medical intervention, such as blood drawing, are quite laborious and are undertaken in our laboratories. For this reason in 2005 the ITR signed a collaborative agreement with AVIS finalizing the implementation of a biobank of voluntary twin donors. AVIS, with its one million blood donors and more than 8 thousand blood transfusion centers, is a well-known organization in Italy. Over the 75 years since its foundation, AVIS has continuously campaigned to educate the public regarding health and solidarity, and it has worked to convince people to give blood on a regular basis without payment, within a system that has become increasingly selective over the years in order to guarantee the maximum safety for both donors and receivers. The protocol agreement between AVIS and ITR covers a series of initiatives that involve the register itself and AVIS, at various levels, in the planning and organization of blood collections from twins willing to donate for transfusion and research purposes. Such initiatives, wherever possible, are carried out in tandem with hospital blood transfusion centers.

Phenotypic data collected with the twin specimens include standardized blood pressure and heart rate, height and weight, waist and hip circumferences, bilateral grip strength, and handedness.

Medical history is recorded, as well as pharmaceutical therapy and smoking and alcohol habits. Hematological standard tests are performed with hepatic enzyme determination, inflammation indices and lipid profiles. Samples are shipped to the ISS Biobank and phenotypic information is entered in the ITR database. The ISS Biobank includes liquid nitrogen tanks with automatic feeding and $-30^{\circ}$ or $-80^{\circ}$ freezers with $\mathrm{CO}_{2}$ backup. Currently, for each twin, several cryovials or paillettes $(0.5-1 \mathrm{ml})$ of plasma, serum, buffy coats and EDTA whole blood are stored in nitrogen vapour phase or at $-80^{\circ}$. Plasma collection 
is made in EDTA and sodium citrate, offering the choice for the most appropriate aliquot in future studies. We are also attempting to store viable cells adding DMSO to whole blood to a final concentration of $10 \%$. Specimens are then slowly cooled to $-80^{\circ}$ at $-1{ }^{\circ} \mathrm{C} /$ minute, and transferred to nitrogen vapour phase.

Following the positive attitude expressed by the young adult cohort, we are planning a large scale, nationwide mail collection of saliva samples. After having purified the samples, high molecular weight DNA will be extracted and stored.

\section{Key Privacy and Ethical Issues}

As mentioned previously, in order to identify twins affected by a certain disease in a multicenter study setting, ITR was used to directly link identifiable personal data records with pathology registers data records held in clinical centers.

Ethical and legal concerns regarding this kind of record linkage arose, in particular after a legislative decree on personal data treatment was issued in 2003 (legislative decree n. 196; 30 June 2003). Taking into account privacy and confidentiality from a legal perspective, together with moral concerns regarding the respect of individuals' and human dignity, it was necessary to structure a new record linkage procedure.

Within this framework, as it was impossible to ask for informed consent from each potential participant, a burdensome task which threatened to postpone the implementation of the studies, a procedure of 'blind' data retrieval (i.e., no patients' personal data were accessed by ITR researchers) has been established. Following the 'case' definition, each clinical center collaborating with ITR creates a file containing records with personal identification variables, one for each case. The center appointed for coordination gathers all the files and structures a 'comprehensive file', having previously checked for redundant information, that is, the presence of the same case in different centers. As it is also important to know about any possible movement of cases from one center to another, new information in this regard is added within each individual record. Finally, a record linkage between the 'comprehensive file' and ITR is performed by a member of technical staff in charge of the center for coordination, in order to match cases and twins. The results of the record linkage produces a list of potential twin cases, after which each clinical center receives communication exclusively about its own cases, currently in treatment or last treated in that center. Specialists in each center contact these individuals and check whether they are twins or not. For those resulting twin cases, the ethical procedure is carried out, the study protocol is described and informed consent requested. Finally, individual personal and clinical data on participants are sent back to the ITR for further contact and epidemiological analyses.

Within the European consortia for genetic epidemiological research (GenomEUtwin, GEHA and
EuroClot), the ITR is faced with the need to institute a research biobank with official recognition and support by the ISS, to be implemented at the National Center for Epidemiology. The establishment of a biobank requires specific guidelines and national rules that are still lacking in Italy. Our twin research framework offered the opportunity to rethink and design new means and procedures for creating a relationship between researchers, citizens (potential participants or patients) and regulating institutional authorities in a national setting.

An official note dealing with the ethical problems associated with the 'storage of blood samples for future studies' was proposed to the local ethical board, to promote the debate on biobanks and also to make the institution more sensitive, and promote willingness to spread a culture of sample donation for research. We worked on structuring a new standard of 'broad' informed consent that emphasizes the rights of donors to freely consent to their (blood/DNA) sample's storage beyond the aims of a specific study, as well as their possible withdrawal at any time, providing a future consent option for further research use (Hansson et al., 2006). Approval was given by the local ethical board, with major ethical concerns for privacy (note that specific regulation on 'genetic data treatment' is still awaiting release by the Privacy Legal Authority), confidentiality and the refusal of the cotwin to provide the sample.

The publicity of constructing an explicit and broadened informed consent was helpful in making individuals, institutions, our local ethical board included, and decision makers more sensitive to the establishment of such research instruments involving human beings' participation. There are arguments against broad consent. These state that the real meaning of an informed consent is violated, and the respect for patients or participants is also neglected (Arnason, 2004). It is our opinion that broad consent together with the requirement for future consent are still ethically sound as the original meaning of informed consent is not violated. Autonomy of individuals and respect for human dignity are guaranteed, provided that: participants risks are not very high, personal information are handled safely, withdrawal from the study as well as from biobank is guaranteed (Helgesson \& Johnsson, 2005) and each new step (i.e., new hypotheses to be proved) is approved by the local ethical board.

The debate on privacy and ethical issues related to the establishment of a biobank from twin blood donors generated an interesting and fruitful discussion about the role that scientists have to play in communicating with a wider audience. It is mandatory for scientists to address, discuss and disseminate scientific, social, and ethical issues to the general public. At the same time, it has become clear that the awareness and understanding of topics and issues which are of concern to the public need to be increasingly raised, if 
we want to generate understanding of genetics, promote public engagement in genetic research and involve people in population genetic studies.

\section{Acknowledgments}

This work was supported by the GenomEUtwin Project (European Union Contract no. QLG2-CT2002-01254) and the EuroClot Project (European Union Contract no. LSHM-CT-2004-005268).

\section{References}

Arnason, V. (2004). Coding and consent: moral challenges of the database project in Iceland. Bioethics, $18,27-49$.

Fraga, M. F., Ballestar, E., Paz, M. F., Ropero, S., Setien, F., Ballestar, M. L., Heine-Suner, D., Cigudosa, J. C., Urioste, M., Benitez, J., Boix-Chornet, M., SanchezAguilera, A., Ling, C., Carlsson, E., Poulsen, P., Vaag, A., Stephan, Z., Spector, T. D., Wu, Y. Z., Plass, C., \& Esteller, M. (2005). Epigenetic differences arise during the lifetime of monozygotic twins. Proceedings of the National Academy of Sciences of the United States of America, 102, 10604-10609.

Hansson, M. G., Dillner, J. D., Bartram, C. R., Carlson, J. A., \& Helgesson, G. (2006). Should donors be allowed to give broad consent to future biobank research? Lancet Oncology, 7, 266-269.

Helgesson, G., \& Johnsson, L. (2005). The right to withdraw consent to research on biobank samples. Medicine Health Care and Philosophy, 8, 315-321.

Nisticò, L., Fagnani, C., Coto, I., Percopo, S., Cotichini, R., Limongelli, M. G., Paparo, F., D’Alfonso, S., Giordano, M., Sferlazzas, C., Magazzù, G., Momigliano-Richiardi, P., Greco, L., \& Stazi, M. A. (2006). Concordance, disease progression, and heritability of coeliac disease in Italian twins. Gut, 55, 803-808.

Ogliari, A., Citterio, A., Zanoni, A., Fagnani, C., Patriarca, V., Cirrincione, R., Stazi, M. A., \& Battaglia, M. (2006). Genetic and environmental influences on anxiety dimensions in Italian twins evaluated with the SCARED questionnaire. Journal of Anxiety Disorders, 20, 760-777.
Priori, R., Medda, E., Conti, F., Cassara, E. A. M., Danieli, M. G., Gerli, R., Giacomelli, R., Franceschini, F., Manfredi, A., Pietrogrande, M., Stazi, M. A., \& Valesini, G. (2003). Familial autoimmunity as a risk factor for systemic lupus erythematosus and vice versa: A case-control study. Lupus, 12, 735-740.

Ristori, G., Cannoni, S., Stazi, M.A., Vanacore, N., Cotichini, R., Alfò, M., Pugliatti, M., Sotgiu, S., Solaro, C., Bomprezzi, R., Di Giovanni, S., Figà Talamanca, L., Nisticò, L., Fagnani, C., Neale, M. C., Cascino, I., Giorni, G., Battaglia, M. A., Buttinelli, C., Tosi, R., \& Salvetti, M. (2006). Multiple sclerosis in twins from Continental Italy and Sardinia. Annals of Neurology, $59,27-34$.

Sarkijarvi, S., Kuusisto, H., Paalavuo, R., Levula, M., Airla, N., Lehtimaki, T., Kaprio, J., Koskenvuo, M., \& Elovaara, I. (2006). Gene expression profiles in Finnish twins with multiple sclerosis. BMC Medical Genetics, 27, 11.

Schousboe, K., Willemsen, G., Kyvik, K. O., Mortensen, J., Boomsma, D. I., Cornes, B. K., Davis, C.J., Fagnani, C., Hjelmborg, J., Kaprio, J., De Lange, M., Luciano, M., Martin N. G., Pedersen, N., Pietilainen, K. H., Rissanen, A., Saarni, S., Sorensen, T. I., Van Baal, G. C., \& Harris, J. R. (2003). Sex differences in heritability of BMI: A comparative study of results from twin studies in eight countries. Twin Research, 6, 409-421.

Silventoinen, K., Sammalisto, S., Perola, M., Boomsma, D.I., Cornes, B., Davis, C., de Lange, M., Harris, J. R., Hjelmborg, J., Luciano, M., Martin, N., Mortensen, J., Nistico, L., Pedersen, N. L., Skytthe, A., Spector, T., Stazi M. A., Willemsen, G., \& Kaprio, J. (2003). Heritability of body height: a comparative study of twin cohorts in eight countries. Twin Research, 6, 399-408.

Stazi, M. A., Cotichini, R., Patriarca, V., Brescianini, S., Fagnani, C., D’Ippolito, C., Cannoni, S., Ristori, G., \& Salvetti, M. (2002). The Italian twin project: From the personal identification number to a national twin registry. Twin Research, 5, 382-386.

The Italian-American Cataract Study Group. (1991). Risk factors for age-related cortical, nuclear, and posterior subcapsular cataracts. American Journal of Epidemiology, 133, 541-553. 